\title{
Intrinsic coordinate system for the tibial plateau
}

\author{
D.P. Piolettia ${ }^{\mathrm{a}, \mathrm{b}, *}$, L.R. Rakotomanana ${ }^{\mathrm{a}, \mathrm{b}}$, P. Dahlgren ${ }^{\mathrm{b}}$, J. Vallotton ${ }^{\mathrm{a}}$, P.-F. Leyvraz ${ }^{\mathrm{a}}$ \\ ${ }^{a}$ Hôpital Orthopédique de la Suisse Romande, Lausanne, Switzerland \\ ${ }^{\mathrm{b}}$ Biomedical Engineering Laboratory, Federal Institute of Technology, Lausanne, Switzerland
}

Accepted 24 July 1997

\begin{abstract}
Determination of the sub-chondral bone density, or more precisely the internal density spot, can be used to evaluate the capability of a knee to sustain normal kinematics. To use this technique as a mean of knee kinematics control, the position of the internal density spot must be determined in a reproducible way. This paper presents a definition of an intrinsic polar coordinate system, allowing to measure the position of the internal density spot of the tibial plateau. Tests of reproducibility gave good results and justify the use of this coordinate system for comparison of the internal density spot position between left and right paired knees. (c) 1998 Elsevier Science B.V. All rights reserved
\end{abstract}

Keywords: Coordinate system; Kinematics; Internal density spot; Reproducibility

\section{Introduction}

Alteration of knee joint kinematics has been shown to modify the distribution of contact pressure between the femoral condyles and the tibial plateau [1]. The sub-chondral bone density of the tibial plateau adapts itself accordingly to this new pressure distribution [2]. This was confirmed by experimental investigations $[3,4]$ which have shown morphologic, metabolic, biochemical and biomechanical changes in the articular knee cartilage of a dog with a transected anterior cruciate ligament.

Anatomically, the tibial plateau is characterized by two spots of higher bone density, corresponding to the load bearing surface of the femoral condyles. Their locations then depend on the knee kinematics. Consequently, this property could be used either to compare the kinematics of right and left paired knees, or to measure the evolution of the gait pattern pathology

\footnotetext{
* Corresponding author. LGM-EPFL, PSE-Ecublens, CH-1015 Lausanne, Switzerland. e-mail: dominique.pioletti@ipa.dp.epfl.ch 0968-0160/98/\$19.00 (c) 1998 Elsevier Science B.V. All rights reserved PII S0968-0160(97)10008-4
}

for one knee. In the first case, at an advanced stage of the gait pathology, the contralateral healthy knee has often served as reference for characterizing the change of kinematics of an injured knee [5]. Comparison of density spot position for the right and the left paired knees has already been used in gait pathology detection in a sheep model [6]. To this end, an empirical coordinate system was proposed to determine the spot position in the tibial plateau for each specimen. However, the geometry of the tibial plateau being different from one specimen to another, it is necessary to assure that the proposed coordinate system is identical for the left and right knee. In the second case, accurate follow-up of spot locations in a knee may be conducted only if the reproducibility of the spot location method is assured.

The aim of this study is hence twofold: (1) to define a coordinate system on the human tibial plateau, intrinsically defined by the specimen geometry; and (2) to assure the reproducibility of such a system and apply it to compare the spot locations on the left and right knees. 


\section{Material and methods}

Twenty Caucasian fresh-frozen anatomical specimen knees (five male pairs and five female pairs, mean age $70.1 \pm 7.1$ years) without radiological evidence of pathology, were retained for the study. The specimens were measured using a high resolution scanner (Densiscan 1000, Scanco Medical Bassersdorf, Switzerland). A transversal slice of $1 \mathrm{~mm}$ wide was immediately derived from the lower bottom of the internal tibial cupule. The density distribution of the sub-chondral tibial plateau is characterized by two spots (one internal and one medial) of high density (Fig. 1A). The position measurement of the spots was performed on the internal one only as it is generally more marked.

\subsection{Intrinsic coordinate system}

Accurate location of the internal spot was achieved by retaining a coordinate system based on inertial properties of the tibial surface of the slice. A polar coordinate system was defined as follows. First, the origin $(O)$ of the system was defined as the centre of gravity of the tibial surface. Second, the axis of maximum moment of inertia of the tibial surface was defined as the zero angle value (reference line). Finally, the internal spot position was defined by its centre of gravity $(G)$. It was located by its distance $(r)$ from the origin $O$ and the angle $(\theta)$ between the line joining $O$ to the internal spot position (dashed line) and the reference line (Fig. 1B).

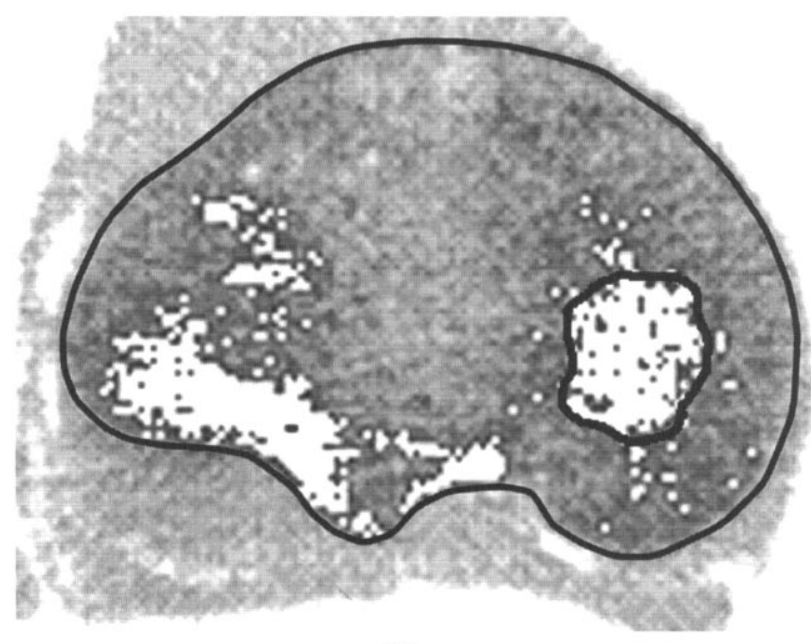

$1 \mathrm{~A}$

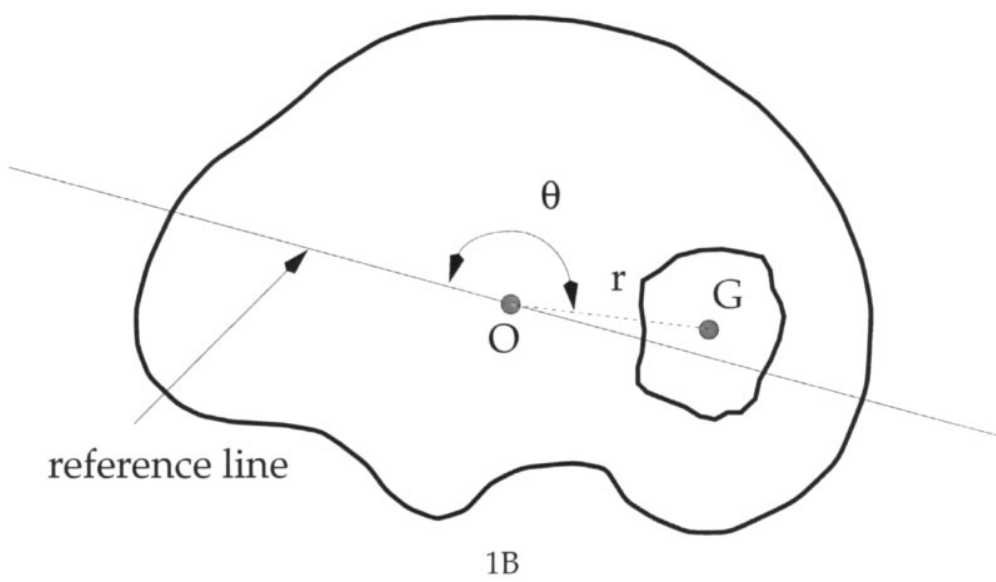

Fig. 1. (A) View of the tibial surface after an image processing which enhanced the contrast between the internal density spot and the tibial plateau. Definition of the tibial plateau and internal spot contours are displayed in black. (B) Definition of the coordinate system of the tibial surface. From the contour definition of the tibial plateau, we obtained its centre of gravity $(O)$ and the axis of maximum moment of inertia (reference line). The internal spot density was defined by its centre of gravity $(G)$. Its position was then located by the distance $r$ (dashed line) between $O$ (taken as the origin of the coordinate system) and $G$ and by the angle $\theta$ between this line and the reference line. 


\subsection{Test of reproducibility}

The reproducibility of the coordinate system was tested in two steps. First, the reproducibility of the moments of inertia was tested. Contouring of the tibial plateau surface and calculation of the area and the moments of inertia were performed three times. This ensures that these values are independent of the way of contouring the tibial surface. Second, the effects of a the slice position on the coordinate system definition was quantified. This aspect is essential whether for comparison of the left and right knee or for a follow-up of the evolution of one knee. It is difficult to scan exactly at the same position for paired knees or during a follow-up. Each knee was hence scanned twice. The first scanner measurement provided the reference values $r_{1}$ and $\theta_{1}$ of the spot coordinates. For the second scanner measurement, we shifted slightly the position $(0.5 \mathrm{~mm})$ of the slice without moving the specimen. The new values $r_{2}$ and $\theta_{2}$ of the spot location were obtained from this new slice position. The resulting differences were calculated between the first and the second measurement.

\subsection{Comparison of right and left knees}

Comparison between paired right and left knees of a same patient constitutes the second basic requirement before applying the method to gait pattern analysis. The comparison of the internal density spot location between left and right paired knees was therefore performed for the reference values $r_{1}$ and $\theta_{1}$.

\section{Results}

\subsection{Test of reproducibility}

Evaluation of the area and of the moments of inertia revealed that the relative differences in the measured values were less than $1 \%$ for each specimen for the three successive measurements. Consequently, the definition of the previous coordinate system has low sensitivity with respect to the determination of the contour. Results corresponding to the measurement of the coordinates $r$ and $\theta$ of the internal density spot in two different slices are shown in Table 1 (male and female specimens). Mean relative differences in coordinate measurements of the internal density spot were $4 \%$ for $r$ and $0.8 \%$ for $\theta$. It was concluded that the proposed coordinate system enables a reproducible position measurement of the internal spot on the tibial plateau.
Table 1

Relative differences between the reference and the second values for $r$ and $\theta$ for all the specimens

\begin{tabular}{llllrl}
\hline $\begin{array}{l}\text { No. male } \\
\text { specimens }\end{array}$ & $r(\%)$ & $\theta(\%)$ & $\begin{array}{l}\text { No. female } \\
\text { specimens }\end{array}$ & $r(\%)$ & $\theta(\%)$ \\
\hline $7 \mathrm{r}_{\mathrm{i}} \mathrm{m}$ & 1.5 & 1.6 & $327 \mathrm{r}_{\mathrm{i}} \mathrm{f}$ & 1.0 & 0.1 \\
$7 \mathrm{gm}$ & 3.3 & 1.0 & $327 \mathrm{lf}$ & 40.0 & 0.9 \\
$311 \mathrm{r}_{\mathrm{i}} \mathrm{m}$ & 2.8 & 1.6 & $331 \mathrm{r}_{\mathrm{i}} \mathrm{f}$ & 1.5 & 1.6 \\
$311 \mathrm{~lm}$ & 2.6 & 0.5 & $331 \mathrm{lf}$ & 2.1 & 0.5 \\
$304 \mathrm{r}_{\mathrm{i}} \mathrm{m}$ & 1.1 & 0.2 & $49 \mathrm{r}_{\mathrm{i}} \mathrm{f}$ & 3.0 & 0.4 \\
$304 \mathrm{~lm}$ & 6.7 & 3.8 & $49 \mathrm{lf}$ & 4.1 & 0.2 \\
$56 \mathrm{r}_{\mathrm{i}} \mathrm{m}$ & 2.9 & 0.5 & $349 \mathrm{r}_{\mathrm{i}} \mathrm{f}$ & 1.1 & 0.4 \\
$56 \mathrm{~lm}$ & 0.1 & 0.2 & $349 \mathrm{lf}$ & 3.8 & 0.05 \\
$322 \mathrm{r}_{\mathrm{i}} \mathrm{m}$ & 0.3 & 0.3 & $340 \mathrm{r}_{\mathrm{i}} \mathrm{f}$ & 1.2 & 0.2 \\
$322 \mathrm{~lm}$ & 1.5 & 2.0 & $340 \mathrm{lf}$ & 0.6 & 0.9 \\
Mean & 2.2 & 1.1 & Mean & 5.8 & 0.5 \\
\hline
\end{tabular}

Notes: $m$, male; $f$, female; $r_{i}$, right; 1 , left.

\subsection{Left and right knee spots}

Location of the internal spot of right and left paired knees was performed for all specimens and the results are presented in Table 2 . It was observed that the mean relative difference between paired knees was $8.2 \%$ for $r$ and $2.2 \%$ for $\theta$.

\section{Discussion}

The abnormal position of the internal density spot on the tibial plateau can be used to reveal pathological knee kinematics. However, such a method is workable only if its position can be measured by means of a reproducible coordinate system, intrinsically defined by the tibial geometry. With this constraint in mind, a polar coordinate system was defined and was based on the moments of inertia of the tibial surface. Moments of inertia were chosen due to their non-sensitivity to the contour determination. This was demonstrated by the low relative differences between successive determinations of these values. This property is probably due to the moments of inertia definition, based on a surface integration. Local variations of the surface delimitation do not sensibly alter the moments of inertia values.

The determination of the internal spot position using the polar coordinate system we proposed was also demonstrated not to be sensitive to the position of the slice on which the measurements were done. This is important since comparison of the spot positions either between left and right paired knees or during an evolution measurement on one knee is likely not to be performed at exactly the same posi- 
Table 2

Relative differences between left and right paired knees for the reference values $r_{1}$ and $\theta_{1}$

\begin{tabular}{lrllrl}
\hline No. specimens & $r(\%)$ & $\theta(\%)$ & No. specimens & $r(\%)$ & $\theta(\%)$ \\
\hline $7 \mathrm{~m}$ & 13.5 & 1.5 & $327 \mathrm{f}$ & 20.0 & 5.7 \\
$311 \mathrm{~m}$ & 6.8 & 1.4 & $331 \mathrm{f}$ & 4.4 & 3.5 \\
$304 \mathrm{~m}$ & 5.1 & 0.1 & $49 \mathrm{f}$ & 1.3 & 1.1 \\
$56 \mathrm{~m}$ & 8.7 & 1.8 & $349 \mathrm{f}$ & 10.2 & 0.01 \\
$322 \mathrm{~m}$ & 6.4 & 4.1 & $340 \mathrm{f}$ & 8.8 & 0.9 \\
Mean & 7.6 & 2.2 & Mean & 8.9 & 2.2 \\
\hline
\end{tabular}

Notes: m, male; f, female.

tion even if remarkable landmarks can be identified. From these tests it can hence be concluded that the proposed coordinate system is reproducible in the determination of the internal density spot position.

Specimens used in this study were assumed to have normal knee kinematics (no radiological evidence of pathology and no ligament injury observed). It can then be stated that normal mean variation of the internal spot density coordinates between pairs of healthy knees was $8.2 \%$ for $r$ and $2.2 \%$ for $\theta$. The proposed coordinate system will be used in a future study such that if the values of $r$ and $\theta$ between left and right knees are much greater than the ones obtained in this study, it will be concluded that a pathological kinematic is present. Especially, in the case of ligament repair after an anterior cruciate ligament, gait analysis study [7] concluded that normal kinematic was restored. It will be interesting to compare this result with the one given by the measurement of the internal spot density position.

\section{References}

[1] Andriacchi TP. Functional analysis of pre and post-knee surgery: total knee arthroplasty and ACL reconstruction. J Biomech Eng 1993;115:575-581.

[2] Noyes FR, Schipplein OD, Andriacchi TP, Saddemi SR, Weise $M$. The anterior cruciate ligament-deficient knee with varus alignment. An analysis of gait adaptations and dynamic joint loadings. Am J Sports Med 1992;20:707-716.

[3] Brandt KD, Myers SL, Burr D, Albrecht M. Osteoarthritic changes in canine articular cartilage, subchondral bone, and synovium fifty-four months after transection of the anterior cruciate ligament. Arthritis Rheum 1991;34:15601570.

[4] Setton LA, Mow VC, Muller FJ, Pita JC, Howell DS. Mechanical properties of canine articular cartilage are significantly altered following transection of the anterior cruciate ligament. J Orthop Res 1994;12:451-463.

[5] McDevitt CA, Muir H. Biochemical changes in the cartilage of the knee in experimental and natural osteoarthritis in the dog. J Bone Jt Surg 1976;58B:94-101.

[6] Muller-Gerbl M, Anetzberger H, Scherer M, Metak G, Putz R, Blumel G. Pathological subchondral bone density patterns after successful reconstruction of the ACL. Proceedings of the combined BORS/EORS meeting, 1994:106.

[7] Timoney JM, Inman WS, Quesada PM et al. Return of normal gait patterns after anterior cruciate ligament reconstruction. Am J Sports Med 1993;21:887-889. 